\title{
Heuristic Algorithm for Anycast Flow Assignment in Connection-Oriented Networks
}

\author{
Krzysztof Walkowiak \\ Chair of Systems and Computer Networks, Faculty of Electronics, Wroclaw University of \\ Technology, Wybrzeze Wyspianskiego 27, 50-370 Wroclaw, Poland \\ Krzysztof.Walkowiak@pwr.wroc.pl
}

\begin{abstract}
Replication of content on geographically distributed servers can improve both performance and reliability of the Web service. Anycast is a one-toone-of-many delivery technique that allows a client to choose a content server of a set of replicated servers. Presenting numerous mirror content servers to a client implies the difficult problem of finding the best server in terms of network performance. We formulate an optimization problem of anycast flows assignment in a connection-oriented network. This is a 0/1, NP-complete problem, which is computationally very difficult due to the size of solution space and constraints. Therefore, we propose computationally effective heuristic algorithm. To our knowledge, this is the first proposal to solve the problem of anycast flow assignment in connection-oriented network. Results of simulations are shown to evaluate performance of proposed algorithm for various scenarios.
\end{abstract}

\section{Introduction}

Web servers providing popular content (MP3 files, movies, electronic books, software distribution) need to scale to a large number of clients. One solution to address this problem is to augment network link capacity or processing resources of the site's server. Another, usually much more cost-effective, solution is to replicate the server or content in many locations in the Internet. This relatively simple technique provides numerous advantages, e.g. inexpensive improvement of client perceived accessibility of content, lower latency, increase of network reliability. Traffic associated with requests to replicas can be modeled as anycast flow. Anycast is a one-to-one-of-many technique to deliver a packet to one of many hosts. One of the most famous techniques that apply anycast traffic is Content Delivery Network (CDN) [1], [3].

Anycast paradigm is expected to be a very attractive approach to give a solution to many important issues that arise in modern computer networks. One of key elements of anycast transmission is the flow assignment problem, i.e. anycast demands should be assigned to network routes in order to optimize a selected network performance function. In this paper we address this problem and propose an efficient heuristic algorithm for static anycast flow assignment. The main novelty of our work is that we focus on connection-oriented (c-o) networks. In c-o network prior to transmitting the data, a virtual connection is established and the data is carried along this connection. Popular c-o techniques are Asynchronous Transfer Mode (ATM), MultiProtocol Label Switching (MPLS). To our knowledge, this is the first algorithm that solves the 
anycast flow assignment problem in c-o network. We consider an existing facility network - we don't optimize location of replica server and network topology.

The anycast non-bifurcated flow assignment (ANBFA) is as follows

Given network topology, traffic demand pattern, location of replica servers, link capacity

Minimize Objective function defined according to information on link flow and

(Maximize) capacity, e.g. network delay, network cost, network survivability

Over selection of replica server, routing (path assignment)

Subject to connection-oriented flow constraints, capacity constraints

The mathematical formulation of the optimization problem can be found in [8], where the ANBFA problem is referred to as CATR (clients' assignment to replicas). Due to limited size of the paper we cannot present detailed information on anycast flow and optimization. Therefore, for more information refer to [1-9].

\section{Anycast Non-bifurcated Flow Deviation Algorithm}

In this section we show Anycast Non-bifurcated Flow Deviation (ANBFD) algorithm for the problem formulated above. ANBFD algorithm is based on the Flow Deviation approach proposed in [2] and widely used for network design problems [4], [7].

In c-o networks an anycast demand consists of two connections: one from the client to the server (upstream) and the second one in the opposite direction (downstream). Upstream connection is used to send user's requests. Downstream connection carries requested data. Let $\delta(i)$ denote index of the connection associated with connection $i$. If $i$ is a downstream connection $\delta(i)$ must be an upstream connection and vice versa. The global anycast non-bifurcated multicommodity flow denoted by $\underline{f^{r}}$ is defined as a vector of flows in all arcs. We call a flow $\underline{f^{r}}$ feasible if for each arc $a$ the flow of $a$ doesn't exceed capacity of $a$.

\section{Algorithm ANBFD}

Let $f^{1}$ denote a feasible anycast flow containing routes for all connections to be established. Let $L(\underline{g})$ denote value of the objective function for a feasible flow $\underline{g}$ and $l_{j}(\underline{g})$ denote a metric of arc $j$. The common approach is to calculate this metric as partial derivative of the objective function over arc flow [2]. We start with $r:=1$. Let $B$ denote a set including connections that have not been already processed. Operator $\operatorname{first}(B)$ returns the index of first connection in set $B$.

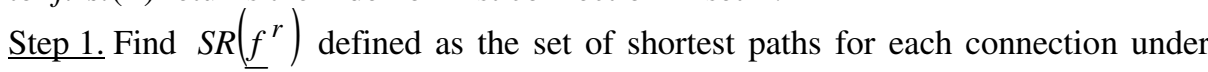
metric $l(f)$. Connections $i$ and $\delta(i)$ associated with one anycast demand are processed jointly. For each node $v$ hosting a replica shortest paths in both directions between $v$ and the second end node are calculated. Finally, we select such a pair of paths for which length of the downstream path is the shortest and add these paths to set $S R\left(\underline{f}^{r}\right)$. Set $B:=P, i:=f i r s t(B)$ and go to step 2 . 


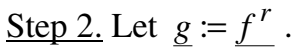

a) Find $\underline{v}$ from $\underline{g}$ by deviating flow of connections $i$ and $\delta(i)$ to the shortest paths included in $S R\left(f^{r}\right)$. Paths for other connections except $i$ and $\delta(i)$ remain unchanged.

b) If $\underline{v}$ is a feasible flow and $L(\underline{v})<L(\underline{g})$ set $\underline{g}=\underline{v}$.

c) If $B=\varnothing$ go to step 3. Otherwise, set $\mathrm{B}:=\mathrm{B}-\{i, \delta(i)\}$ and go to step 2a.

$\underline{\text { Step 3. If } g}=f^{r}$ stop the algorithm, since the solution cannot be improved. Otherwise, set $r:=r+1, \underline{f}^{r}:=\underline{g}$ and go to step 1 .

In order to find feasible starting solution we can apply an algorithm based on the initial phase of the FD algorithm [2], [7]. One of the main advantages of ANBFD is that it takes into account all possible routes in the network and the calculation time of ANBFD doesn't depend on the number of candidate routes. Therefore, there is no need to reduce size of the problem given by the number of routes. Algorithm ANBFD converges in finite number of steps. However, the number of steps and consequently algorithm complexity depends on particular parameters of the considered problem (network topology, location of replicas, demand matrix).

\section{Results}

The test network consists of 36 nodes and 144 directed links [9]. We run experiments for various scenarios of replica location and number of replicas. Since more data is received by clients, than is sent to replicas. Therefore, we assume that upstream bandwidth is 0.1 of the downstream bandwidth. The total demand is calculated as a sum of all downstream and all upstream bandwidth requirements.

In many real life cases, a client is assigned to the closest replica in terms of the hop number. Therefore, for comparison we develop a simple heuristic, which is referred to as CR (Closest Replica). Algorithm CR assigns each anycast demand to the closest (in terms of hop number) replica. This way we obtain a unicast non-bifurcated flow assignment problem, which is solved by a non-bifurcated f FD algorithm.

We assume that the objective function represents the total flow in the network. In Table 1 we report results obtained for algorithms ANBFD and CR for the different number and locations of replica servers. Empty cells indicate that for a particular case the algorithm cannot find a feasible solution. First, quite intuitive, observation is that increasing the number of replicas decreases the network flow. Moreover, if there are more replicas in the network, demands with higher bandwidth requirements can be satisfied. If we compare ANBFD against CR, we can see that for relatively low loads both algorithms perform similarly. This can be explained by the fact that for low congestion each demand is assigned to nearest replica for both algorithms. The major advantage of ANBFD is for the case with 4 replicas - for two highest loads CR cannot find a feasible solution, while ANBFD can. This follows from the construct of ANBFD, which can reroute anycast demand to another replica, what enables selection of less congested links. Similar results were obtained for other tests. 
Table 1. Performance of algorithms ANBFD and CR for various replica location and demands

\begin{tabular}{|c|c|c|c|c|c|c|c|c|}
\hline \multirow{2}{*}{$\begin{array}{c}\text { Total } \\
\text { Demand }\end{array}$} & \multicolumn{2}{|c|}{1 replica in (30) } & \multicolumn{2}{c|}{2 replicas $(9,23)$} & \multicolumn{2}{c|}{3 replicas in $(9,23,30)$} & \multicolumn{2}{c|}{$\begin{array}{c}4 \text { replicas in } \\
(5,9,23,30)\end{array}$} \\
\cline { 2 - 9 } & ANBFD & CR & ANBFD & CR & ANBFD & CR & ANBFD & CR \\
\hline 1080 & 3088 & 3088 & 2125 & 2125 & 1722 & 1700 & 1362 & 1370 \\
\hline 3105 & 9281 & 9425 & 5945 & 5945 & 4834 & 4805 & 3859 & 3885 \\
\hline 5040 & & & 10502 & 10430 & 7886 & 7890 & 6331 & 6400 \\
\hline 7020 & & & & & 11450 & 11515 & 8818 & 8915 \\
\hline 9000 & & & & & & & 12225 & 11925 \\
\hline 10980 & & & & & & & 14861 & \\
\hline 11880 & & & & & & & 16146 & \\
\hline
\end{tabular}

\section{Final Remarks}

The heuristic algorithm ANBFD proposed in this work is, according to our best knowledge, the first attempt to optimize anycast flows in connection-oriented networks. Numerical experiments have been conducted to evaluate performance of the algorithm for various scenarios. Results have confirmed that introducing new content servers enables substantial reduction of network flow what, consequently, decreases network delay and improves network reliability. The experiment shows that ANBFD is more efficient than the $\mathrm{CR}$ in solving relatively higher congested problems.

Acknowledgements. This work was supported by a research project of the Polish State Committee for Scientific Research carried out in years 2005-2007.

\section{References}

1. Awerbuch, B., Brinkmann, A., Scheideler C.: Anycasting in adversarial systems: routing and admission control. Lecture Notes in Computer Science, LNCS 2719 (2003), 1153-1168

2. Fratta, L., Gerla, M., Kleinrock, L.: The Flow Deviation Method: An Approach to Storeand-Forward Communication Network Design. Networks Vol. 3 (1973) 97-133

3. Hao, F., Zegura, E., Ammar, M.: QoS routing for anycast communications: motivation and an architecture for DiffServ networks. IEEE Communication Magazine, 6 (2002), 48-56

4. Kasprzak, A.: Designing of Wide Area Networks. Wroclaw Univ. of Tech. Press, (2001)

5. Partridge, C.,Mendez, T., Milliken, W.: Host Anycasting Service. IETF RFC 1546 (1993)

6. Peng, G.: CDN: Content Distribution Network. Technical Report (2003)

7. Pióro, M., Medhi, D.: Routing, Flow, and Capacity Design in Communication and Computer Networks. Morgan Kaufman Publishers (2004)

8. Walkowiak, K.: An exact algorithm for design of content delivery networks in MPLS environment. Journal of Telecommunications and Information Technology 2 (2004), 13-22

9. Walkowiak, K.: QoS Dynamic Routing in Content Delivery Networks, to appear in Proceedings of Networking 2005 